\title{
New aspects of the vulnerability stress model in general, its relevance in schizophrenic psychoses and the place of antipsychotics
}

\author{
Hans-Jürgen Möller ${ }^{1}$
}

Published online: 19 July 2017

(c) Springer-Verlag GmbH Germany 2017

Originally, the vulnerability stress model was developed in the context of schizophrenic psychoses to give a better insight into the complexity of the multifactorial etiopathogenesis of these disorders and to provide a roadmap for different kinds of interventions in terms of psychopharmacological and psychosocial treatments $[2,5,10,11]$. Later on it seemed adequate to extend this conceptual approach to other mental disorders.

In the current issue of EAPC, several papers are related to stress in its diverse manifestations under different conditions and in the context of different mental disorders like schizophrenia (Mihaljevic et al. 2017), depressive disorders (Tollenaar et al. 2017), anxiety disorders (Chin et al. 2017), posttraumatic stress disorder (Cwik et al. 2017) and even stroke (Kronenberg et al. 2017). The authors in their interesting contributions look from different angels on this general topic. This involves clinical perspectives, different neurobiological aspects like genetic variants (FKBP5) in the context of the stress-related HPA-axis-alteration, the role of polymorphisms of the oxytocin gene in the context of early life stress or the description of fMRI alterations in different brain regions. Altogether, these articles forward a better understanding of the complexity of psychosocial and neurobiological factors and underpinnings of mental disorders, which is not mirrored by the clinical classification systems like ICD and DSM-5 [7].

This also applies to the psychopharmacological treatments we can offer our patients with stress-related disorders. Most of these are treated with-according to their

Hans-Jürgen Möller

Hans-Juergen.Moeller@med.uni-muenchen.de

1 Department of Psychiatry, University of Munich, Munich, Germany licensed indications—-so-called traditional compounds like antidepressants, antipsychotics, anxiolytics or in the new nomenclature [9] more specifically according to their main pharmacological properties: serotonin/norepinephrine reuptake inhibitors, serotonin reuptake inhibitors, alpha- 2 receptor antagonists, dopamine receptor antagonists, dopamine serotonin antagonists, benzodiazepine receptor agonists, etc. Considering these pharmacological mechanisms allows a more extended use related to syndromal or symptomatic aspects, independent from classificatory clusters [8, 12]. All the pharmacological mechanisms mentioned above apparently involve-among others-also stress-related properties. We thus hope on the development of modern compounds with more specific modes of actions.

Especially due to side-effects, adherence can become a problem in single patient, additionally a certain group of compounds can be criticized. Currently, latter is the case with antipsychotics, traditionally also called neuroleptics, in modern terminology the dopamine antagonists and the dopamine serotonin antagonists. These compounds have proven efficacy in reducing positive symptoms in acute schizophrenic episodes plus in the risk of relapses, and to a lesser degree in negative symptoms [8]. Part of their efficacy, especially the relapse preventive capacity, formerly was explained in the frame of the vulnerability stress model. All actually available evidence from randomized control group studies indicates that the long-term medication with antipsychotics substantially reduces the risk of relapse. Alternative strategies like low dose treatment or intermittent treatment with antipsychotics did not reach a comparable level of efficacy. Thus, relapse prevention with continuous long-term use of antipsychotics is seen since longtime as the EBM state of the art, recommended in most guidelines.

In recent years, as already sometimes in the past, a discussion on the necessity of principal relapse prevention and an 
optimal treatment strategy in general restarted. This discussion was inspired by results from recent (mostly naturalistic) MRI-studies in which patients not under continuous antipsychotic long-term treatment showed positive outcomes. Especially findings on a possible association of continuous long-term treatment with antipsychotics and volume reductions in specific brain areas-again mostly from naturalistic follow up studies-heated up this discussion. Recent metaanalyses underlined this association more or less consistently, but failed in proving a causal relationship [4].

Interpretation of the pathogenetic relationship is difficult, given the available data is: naturalistic. It must be considered that MRI-measured brain alterations are manifest already before the first psychotic episode, thus being an indicator of the neurodevelopmental disorder and basis for the increased vulnerability. These brain alterations can increase before the first psychotic episode and further proceed in the long-term course of the illness with or without antipsychotic treatment, especially when associated with negative symptoms $[3,6]$. Altogether, the relationship between brain alterations and course of the illness is very complex and not easy to disentangle, especially not via naturalistic studies. There, however, are hints, although inconsistent, that brain volume reduction is stronger associated with so-called first generation antipsychotics (dopamine receptor antagonists) than with second generation antipsychotics (dopamine serotonin receptor antagonists). Anyhow, all this should not neglect the principal problem [4]. If we consider that already quite simple motoric activities can induce MRI-measured brain volume alterations [1], in this case brain volume increase, why should antipsychotics interfering with several cerebral processes not have this brain volume reducing effect? The question then is rather, what kind of consequences are associated with these brain alterations? Besides, it has principally to be stated that brain volume reduction associated with antipsychotic treatment is not the result of loss of neurons [4], but of a reduction of the neuropil, dendrites, etc.

Considering the data of animal studies, we are confronted with results indicating a neurotoxic effect of antipsychotics, especially of first generation antipsychotics (dopamine receptor antagonists), while second generation antagonist (dopamine serotonin antagonists) seem somewhat more benign in this respect [4]. However, the animal data, although experimental, are by far not conclusive. In addition, a naïve transfer of animal study results on the human section could lead to false consequences. This is especially true for the transfer into individuals suffering from schizophrenic psychoses. It seems conceivable, that antipsychotics can negatively influence the brain of healthy people, but perhaps demonstrate protective effects on the brain of individuals suffering from schizophrenia.

We thus are far away from being able to draw firm conclusions. The issue urgently needs further research, both in animal plus carefully designed human studies. For the moment it should be stated, that apparently there is no clear evidence of volume reduction associated with antipsychotic long-term treatment to be associated with a reduction of cognitive capacities. Thus, we should currently not prematurely change the traditional strategy based on the vulnerability stress model and for the time being retain the continuous long-term treatment as the best approach to reduce the risk of relapse. However, we should keep in mind to prescribe our patients the individually adjusted lowest possible dose. This could be optimized by precisely predicting the patients at high risk of a relapse and those without any risk or those not profiting from relapse prevention by antipsychotics (non-responder). Regrettably, we currently are still missing sufficiently powerful predictors like MRI or blood related biomarkers, for which reason we carry on including all patients in all therapeutic options, although for the afore mentioned patients relapse prevention with antipsychotics probably is inefficient.

\section{References}

1. Falkai P, Malkow B, Wobrock T et al (2013) The effects of physical exercise in schizophrenia and affective disorders. Eur Arch Psychiatry Clin Neurosci 263(6):451-467

2. Falkai P, Möller HJ (2012) Psychopathology. Genetics and the stress vulnerability hypothesis. Eur Arch Psychiatry Clin Neurosci 262(3):181-182

3. Galderisi S, Merlotti E, Mucci A (2015) Neurobiological background of the negative symptoms in schizophrenia. Eur Arch Psychiatry Clin Neurosci 265(7):i543-i558

4. Goff D, Falkai F, Fleischhacker W et al (2017 ) AJP 2017 psychiatry online.org

5. Halanova E, Haralanow S, Beraldi A et al (2012) Subjective emotional over-arousal to neutral social scenes in paranoid schizophrenia. Eur Arch Psychiatry Clin Neurosci 262(1):59-68

6. Koutsouleris N, Vavatzikos C, Borgwardt S et al (2014) Accelerated brain aging and beyond: a neuroanatomical marker of psychiatric disorders. Schizophr Bull 40(5):1140-1153

7. Möller HJ, Bandelow B, Bauer M et al (2015) DSM-5 reviewed from different angles: goal attainment, rationality, use of evidence, consequences.: general aspects and paradigmatic discussion of depressive disorders. Eur Arch Psychiatry Clin Neurosci 265(2):87-106

8. Möller HJ, Czobor P (2015) Pharmacological treatment of negative symptoms in schizophrenia. Eur Arch Psychiatry Clin Neurosci 265(7):567-578

9. Möller HJ, Schmitt A (2016) Neuroscience based nomenclature $(\mathrm{NbN})$ to replace traditional terminology of psychotropic medications. Eur Arch Psychiatry Clin Neurosci 266(5):385-386

10. Moritz S, Köther U, Hartmann M et al (2015) Stress is a bad advisor. Stress primes poor decision making in deluded psychotic patients. Eur Arch Psychiatry Clin Neurosci 265(6):461-469

11. Ayub M, Saeed K, Kingdon D et al (2015) Rate and predictors of psychotic symptoms after Kashmir earthquake. Eur Arch Psychiatry Clin Neurosci 265:471

12. Schennach R, Riedel M, Obermeier M et al (2015) What are residual symptoms in schizophrenia? Clinical description and 1-year persistence within a naturalistic trial. Eur Arch Psychiatry Clin Neurosci 265(2):107-116 